\title{
IOT ENABLED INDOOR NAVIGATION SYSTEM DESIGN FOR EMERGENCIES
}

\author{
A. Duru ${ }^{1}$, I. R. Karas ${ }^{2, *}$ \\ ${ }^{1}$ Dept. of Biomedical Engineering, Karabuk University, 78050 Karabuk, Turkey - andayduru @karabuk.edu.tr \\ ${ }^{2}$ Dept. of Computer Engineering, Karabuk University, 78050 Karabuk, Turkey - ismail.karas@karabuk.edu.tr
}

Commission VI, WG VI/4

KEYWORDS: IoT, UWB, Indoor Navigation System, Fall Detection, Wireless Sensor Network, Remote Sensing.

\begin{abstract}
:
Global Navigation Satellite System is widely accepted and it has good positioning accuracy for outdoor applications. However, it does not provide sufficient positioning accuracy for inside of buildings. Signals are attenuated inside of buildings or dense high building areas because various materials between satellites and receiver create interference to the signal propagation. Thus, indoor positioning technologies have emerged to cover the problem. In this paper, indoor positioning technologies have been investigated and UWB based indoor positioning technology has been chosen between them. In addition to indoor positioning, accelerometer and Wi-Fi connection has been added to the system for mobility. The accelerometer detects emergency cases and alerts relatives of an intended person by using web service. Hereby, remote navigation system design is presented for first aid of the user. The system can detect emergencies, especially falls using the accelerometer and it can locate patients anywhere in the world. The designed system showed that it is possible to detect emergencies and transfer to the web with a $24.17 \mathrm{~cm}$ average positioning error. Also, it is an assuring system for future remote positioning applications.
\end{abstract}

\section{INTRODUCTION}

The internet revolution has started to take place in every aspect of our lives. Cell-phones, home appliances, cities, farms, buildings, roads, and cars are equipped with more sensors day by day. Increasing number of sensors allows devices to sense the physical world and act accordingly. Also, the revolution enables us to manage remote applications. For instance, agriculture takes advantage of IoT. Using sensing equipment, drones can easily find driest parts of the farming field. It gives the opportunity of using water resources efficiently and economically. Also, drones can scan the ground and spray the correct amount for fighting pest (Bacco, M. et al., 2018). Outdoor robots are also useful for cleaning purposes in cities. Remote mobile robot positioning can help when performing military and civilian tasks in urban areas (Zhang, J., 2011). For example, trash can be collected with outdoor service robots (Nishida, T. et al., 2006). Remote applications can ease and increase the efficiency of work. This type of outdoor applications uses the Global Navigation Satellite System (GNSS) signals for positioning. Outdoor navigation requirement for remote sensing applications has been solved with the increase in GNSS accuracy. The working principle of the system can be explained by calculation of radio signal travel time between satellites and receiver. However, GNSS signals are not accurate for indoor positioning systems because of the non-line of sight propagation. Since signal travel time is one of the most important factors to give correct information data to the user, materials between satellites and user cause interference to signal propagation. Thus, the accuracy drops considerably. Therefore, an indoor navigation system (INS) is required to overcome this issue. Smart buildings with INS can enable real-time direction maps, self-positioning robots, personnel and equipment tracking applications, statistics of most visited shops, security applications and so on.

Variety of signals and propagation mechanisms have been developed and discussed by researchers to make reliable INS.
Different indoor positioning technologies can be classified with respect to its working principle. Radio Frequency Identification (RFID), Wi-Fi, Bluetooth, ZigBee, Ultrasonic, Ultra-Wideband based Radio Frequency (RF) and camera, Inertial Measurement Unit (IMU), Visible Light Communication (VLC) based solutions are researched.

RFID tags can be classified by the method of powering them. Active tags include battery and it powers the circuit. By this way, it is possible to send high-level signals as a response of low-level reader signals. Received Signal Strength Indicator (RSSI) is the measure of distance in this technology. There are various problems with radio wave propagation in indoor environments. For example, absorption, diffraction, rare LOS path and reflection (Bouet, M. et al., 2008). Therefore, environmental factors such as people, objects, walls affect RSSI value. On the other hand, a passive tag does not have an internal battery. Predefined points are used to track location for seeking point. Although they are cheap in price, their use can be advantageous in some cases. Semi-passive tags are similar to passive tag, but the main difference is that the battery is not used for generating a response message. Powering the internal circuitry which is not related to positioning is the reason to have an internal battery. Specific item or equipment tracking with given parameters is easier to apply for this technology.

$\mathrm{Wi}-\mathrm{Fi}$ is a radio-frequency based positioning system and system measures RSSI to measure distance value. Although it has diverging advantages such as high bandwidth, different frequency bands $(2.4,3.6,5 \mathrm{GHz})$ and adoption, there are also disadvantages as the same with RSSI based positioning systems. Since most of the mobile devices already have built-in Wi-Fi sensors, extra hardware cost will be minimum. However, accuracy is very limited with this positioning technology. Therefore, the system needs extra algorithms or hardware to make it good enough to use (He, S., et al., 2016). A hybrid system can be considered Wi-Fi with GNSS and it can be used to get both indoor and outdoor location data.

\footnotetext{
*corresponding author.
} 
Bluetooth estimates the position of the tag in similar ways with Wi-Fi, but it uses different frequency band (2.4Ghz). RSSI value is the estimation method and mentioned disadvantages also apply for Bluetooth based positioning systems. Most of the cell phones have built-in Bluetooth sensors inside. Thus, there will be no extra installation cost for navigation. However, reference points must be installed for positioning algorithm to compute. Bluetooth technology has better resolution than Wi-Fi (Neburka, J. et al., 2016). As navigation area increases, multiples of Bluetooth reference points must be placed for better accuracy.

On the other hand, positioning system with ZigBee technology also stands on RSSI measurements. The issue for Radio Frequency (RF) signal behavior applies for this technology. It operates in the $2.4 \mathrm{GHz}$ frequency band. One of the papers proposed that average absolute error can be under $3 \mathrm{~m}$ with dynamic calibration (Larranaga, J. et al., 2010). Since RSSI value can vary considerably with environmental factors, it is better to use for home automation, industrial automation, smart monitoring applications because of the power consumption rate, network size, and network architecture. Table 1.1 compares available active RSSI based technologies.

Table 1.1. Comparison of active RSSI based technologies (Proskochylo, A. et al., 2015).

\begin{tabular}{l|l|l|l}
\hline & Wi-Fi & Bluetooth & ZigBee \\
\hline Protocol & 802.11 & 802.15 .1 & 802.15 .4 \\
\hline $\begin{array}{l}\text { Power } \\
\text { Consumption }\end{array}$ & High & Low & Very Low \\
\hline Range & $100 \mathrm{~m}$ & $10 \mathrm{~m}$ & $10-100 \mathrm{~m}$ \\
\hline Bandwidth & $22 \mathrm{MHz}$ & $1 \mathrm{MHz}$ & $\begin{array}{l}0.3 / 0.6 \quad \mathrm{MHz} \\
-2 \mathrm{MHz}\end{array}$ \\
\hline Network Size & 2007 & 8 & $>65000$ \\
\hline $\begin{array}{l}\text { Application } \\
\text { Focus }\end{array}$ & Speed & $\begin{array}{l}\text { Cable } \\
\text { Replacement }\end{array}$ & Automation \\
\hline R
\end{tabular}

In the ultrasound-based method, ultrasonic sound waves are sent by a transmitter and then the signals are picked up by a receiver node. Time of Flight(TOF) data is used for distance estimation. Measurement with ultrasonic waves can achieve millimeter accuracy with a range of $6 \mathrm{~m}$. Unfortunately, undesirable phenomena can affect ultrasonic waves such as crosstalk, echo, and jamming (Medina, C. et al., 2013, De Angelis, G. et al., 2015)

Large bandwidth signals allow transmission of short duration high energy pulses. Since it is possible to sense pulses as high as 20 times in a second, TOF and time of arrival positioning techniques can be applied. Therefore, high time resolution improves the accuracy of indoor positioning systems. Thus, it is possible to reach very good accuracy (Quyum, A., 2013). As a result of the large spectrum, Ultra-Wide Band (UWB) signals can penetrate through objects such as walls, doors etc. Since the energy of the signal is dispersed over a broad frequency range, the time frame can be kept compact without exceeding the energy density limits which in turn compresses the edge used for determining the receive time, allowing for very accurate time-stamping of incoming messages. High energy signals provide excellent scalability, and the technology offers reduced complex processing of the obtained results (Dadeby, S., 2017). The range and offered accuracy give very promising results for indoor positioning algorithms. On the other hand, UWB chips are not implemented on mobile devices and they are relatively expensive products. As the chips are produced in large scales, they will meet the indoor positioning needs for various industries.

Image processing is another technique for INS application. Since existing cameras can acquire images, data on those images are useful to differentiate objects or person. Also, the tag does not have to carry any circuit for tracking. Image processing is required to measure distance and complex environments require complex algorithms to detect the target. A larger field of view and greater pixel density gives better data for processing. However, there are some issues about the camera-based positioning systems. A tracked person should be in the line of sight for processing, otherwise, the image is useless. Therefore, multiple cameras should be installed to increase accuracy and line of sight. The deployment price gets higher as the number of cameras increases. Another problem is a privacy concern. Tracking and recording image has a potential of unethical behavior, so it is undesirable for public applications.

Visible light can also be used for a data transaction, so there are a variety of application areas such as security, body area networks, and indoor navigation applications. This technology uses visible light between $375-780 \mathrm{~nm}$ for data transaction. The system works by detecting coded emitted light. The stationary emitters send a message including position data by blinking and target can acquire the location of itself. However, a line of sight is needed for this application and extra hardware should be deployed.

Dead reckoning technique uses IMU which includes accelerometer, gyroscope, and magnetometer for positioning. The last known position is combined with the accelerometer and angular velocity data and the new position is estimated. Since every position change depends on the previous data, the error is cumulative. Thus, frequent calibration is needed for this application. IMU data is better to use in hybrid systems for increasing the accuracy of positioning technologies.

This paper not only focuses on indoor navigation systems but also IoT application for telecare system. Ultra-Wideband technology has been chosen among the mentioned positioning techniques because of its advantages over the other technologies. The main features of the technology provide low power consumption rate, low interference from other electronic devices, robustness and precise measurements. Onboard accelerometer is responsible for emergency cases by checking acceleration data in each second. By this way, urgent falls can be detected. On the other hand, IoT enabled design gives an opportunity to communicate with the intended person or department. Overall, the designed system can draw data from accelerometers to find out emergency cases and provide information about the case with position data for further assistance.

\section{SYSTEM DEVELOPMENT}

The design of the system includes UWB module communication for estimating distance, position calculations and sending navigation and emergency case to the web server.

\subsection{Distance calculation with UWB modules}

The sensor has high timing accuracy because of the high frequency. Therefore, it can be used in two-way ranging systems for positioning. The two-way ranging concept can be explained by the data acquisition of sensors. Measuring time of flight signals between sensors gives data about the distance between them. The distance between two objects can be calculated by multiplication of speed of radio waves and flight 
time of the signal. Correct timestamping directly affects the accuracy of the system. Timing is progressed by the internal circuit of UWB modules (DWM1000). Timestamping between radio messages allows us to find the location of the tag and it is known as two-way ranging. The two-way ranging concept is explained in more detail in (Duru A. et al., 2018).

\subsection{Positioning and Fall Detection Algorithms}

There are a variety of algorithms to calculate position; proximity algorithm, triangulation algorithm and fingerprinting algorithm. Connectivity principle is used in a proximity algorithm. After closest point connection, the relative position is determined by the power of RSSI. Accuracy is directly related to the number of anchors. In the triangulation algorithm, calculation of the position is handled by using geometry. It can be classified in two subdivisions as lateration and angulation. Firstly, the travel time of the signal is received by lateration algorithm and it needs at least three anchors to locate tag. Also, there need to be $N+1$ sensors for $N$-dimensional places. Secondly, the angulation algorithm uses the angle of arrival signals. There must be a well placed array of antennas to apply this type of positioning algorithm. The other positioning algorithm is based on map-matching. Map-matching algorithm collects signal data and creates a database. This database comprises training phases by matching real coordinates and signal characteristics. The second phase locates by measuring signal values and searches the database for nearest points. On the other hand, it has training problems due to unstable signals (Shchekotov, M., 2015).

In this study, tag position has been calculated via UWB radio modules with three reference points. After time-based distance measurements from each reference points, trilateration has been chosen for position calculation of tag. In trilateration, the unknown point $(\mathrm{x}, \mathrm{y})$, known points $\left(\mathrm{x}_{\mathrm{i}}, \mathrm{y}_{\mathrm{i}}\right)$ and radius of the circle $\left(r_{i}\right)$ are expressed in three sets of circle equations with known radius parameter and reference positions (Eq. 1).

$$
\begin{aligned}
& \left(x-x_{1}\right)^{2}+\left(y-y_{1}\right)^{2}=r_{1}^{2} \\
& \left(x-x_{2}\right)^{2}+\left(y-y_{2}\right)^{2}=r_{2}^{2} \\
& \left(x-x_{3}\right)^{2}+\left(y-y_{3}\right)^{2}=r_{3}^{2}
\end{aligned}
$$

Expanding out squared parameters and subtracting equations from each other yields a solution with two unknowns $(\mathrm{x}, \mathrm{y})$. Microcontroller-unit(MCU) (ATMega328p) has been programmed to solve the given equations regarding positions of reference points and distance measurements.

Accelerometer (ADXL345) has been chosen to monitor falls of a person. Adjusting threshold value for the algorithm establishes a fall alert system. The sensor has a high resolution (13-bit) and it is a small, thin, ultralow power as low as $23 \mu \mathrm{A}$ device (ADXL345-EP datasheet). Since the system needs an alert for emergency cases, accelerometer provides information about if there are any injuries happened or not. Then, MCU sends an output message to the Wi-Fi module about position data and emergency case.

Wi-Fi module (ESP8266) has been programmed to take input from MCU and send the navigation and emergency data to the cloud. The web server has been prepared to show input data as a final part of the IoT application.

\section{RESULTS}

The structure of the web-page includes position data, fall detection case and activity parts. The algorithm constantly checks accelerometer data for an emergency case instead of calculating position data for each second. Therefore, the program will not loop all the iterations. If fall is detected, position data is updated in the web-page with case information. Fig.2.1. shows the developed web-page while the system is alerting.

\section{Position Data and Case}

$\begin{array}{ccc}\text { Position Data } & \text { Case } & \text { Activity } \\ \mathrm{X}-2.2 \mathrm{~m} & \text { Fall Detected } & \text { No } \\ \mathrm{Y}-3.1 \mathrm{~m} & & \end{array}$

Fig.2.1. Web Navigation and Emergency Service

Laser meter has been used for correct position of tag. After 5 different emergency trials, the average error of the application is calculated as $24.17 \mathrm{~cm}$ and the standard deviation is $4.52 \mathrm{~cm}$.

\section{CONCLUSION}

In this study, indoor positioning technologies are researched and UWB technology gave promising results for future indoor positioning applications with good accuracies. Designed system can achieve positioning under $30 \mathrm{~cm}$ error. The system accuracy is very promising when compared to other technologies. Updating frequency can be lowered to increase battery performance to acquire long-term measurement data. On the other hand, IoT enabled to access data all around the world, this application provides telecare data for relatives of the intended person. The application can be extended with different positioning and filtering algorithms for more accurate results. Other types of body posture data can be acquired and specific cases can be detected with additional sensors.

\section{REFERENCES}

Bacco, M., Berton, A., Ferro, E., Gennaro, C., Gotta, A., Matteoli, S., Paonessa, F., Ruggeri, M., Virone, G. and Zanella, A., 2018 IoT Vertical and Topical Summit on Agriculture Tuscany (IOT Tuscany), https://doi.org/10.1109/IOTTUSCANY.2018.8373043.

Zhang, J., Autonomous Navigation for an Unmanned Mobile Robot in Urban Areas, Proceedings of the 2011 IEEE International Conference on Mechatronics and Automation $\begin{array}{lllll}\text { August } & 7 & - & 10, & \text { Beijing, }\end{array}$ https://doi.org/10.1109/ICMA.2011.5986288.

Nishida, T., Takemura, Y., Fuchikawa, Y., Kurogi, S., Ito, S., Obata, M., Hiratsuka, N., Miyagawa, H., Watanabe, Y., Suehiro, T., Kawamura, Y. and Ohkawa, F., SICE-ICASE International Joint Conference 2006 Oct. 18-21, 2006 in Bexco, Busan, Korea, https://doi.org/10.1109/SICE.2006.315055.

Bouet, M. and Dos Santos, A. L., RFID tags: Positioning principles and localization techniques, 1st IFIP Wireless Days, WD 2008, https://doi.org/10.1109/WD.2008.4812905. 
He, S. and Chan, S. H. G., Wi-Fi fingerprint-based indoor positioning: Recent advances and comparisons, IEEE Communications Surveys And Tutorials, 18 (1): 466-490, https://doi.org/10.1109/COMST.2015.2464084.

Neburka, J., Tlamsa, Z., Benes, V., Polak, L., Kaller, O., Bolecek, L., Sebesta, J., and Kratochvil, T., Study of the performance of RSSI based Bluetooth smart indoor positioning, 2016 26th International Conference Radioelektronika, Radioelektronika 2016, 121-125, https://doi.org/10.1109/RADIOELEK.2016.7477344.

Larranaga, J., Muguira, L., Lopez-Garde, J. M., and Vazquez, J. I., An environment adaptive ZigBee-based indoor positioning algorithm, 2010 International Conference On Indoor Positioning And Indoor Navigation, IPIN 2010 - Conference Proceedings, $15-17$, https://doi.org/10.1109/IPIN.2010.5647828.

Proskochylo, A., Vorobyov, A., Zriakhov, M., Kravchuk, A., Akulynichev, A., and Lukin, V., Overview of wireless technologies for organizing sensor networks, 2015 2nd International Scientific-Practical Conference Problems Of Infocommunications Science And Technology, PIC S And T 2015 - Conference Proceedings, 39-41, https://doi.org/10.1109/INFOCOMMST.2015.7357263.

Medina, C., Segura, J. C., and De la Torre, Á., Ultrasound indoor positioning system based on a low-power wireless sensor network providing sub-centimeter accuracy, Sensors 13(3): 3501-3526, https://doi.org/10.3390/s130303501.
De Angelis, G., De Angelis, A., Moschitta, A., and Carbone, P., Ultrasound-based positioning using Time of Flight measurements and crosstalk mitigation, Conference Record IEEE Instrumentation and Measurement Technology Conference, $2015 \quad-\quad$ July, $1865-1870$, https://doi.org/10.1109/I2MTC.2015.7151565.

Quyum, A., "Guidelines for Indoor Positioning", M.S. Thesis, Lulea University of Technology, Lulea, Sweden, (2013).

Dädeby, S., A system for indoor positioning using ultrawideband technology, M.S. Thesis, University of Gothenburg, Gothenburg, Sweden, (2017).

Duru, A., Sehirli, E., Kabalci, I., Ultra-Wideband Positioning System Using TWR and Lateration Methods, ICEMIS '18 Proceedings of the Fourth International Conference on Engineering \& MIS 2018, Istanbul, https://doi.org/10.1145/3234698.3234756.

Shchekotov, M., Indoor localization methods based on Wi-Fi lateration and signal strength data collection, Conference Of Open Innovation Association, FRUCT, 2015-June, 186-191, https://doi.org/ 10.1109/FRUCT.2015.7117991.

ADXL345-EP datasheet, Analog devices, https://www.analog.com/media/en/technicaldocumentation/data-sheets/adx1345-ep.pdf (Last access 29 October 2018) 